\title{
Solutions of Konopelchenko-Dubrovsky Equation by Traveling Wave Hypothesis and Lie Symmetry Approach
}

\author{
Sachin Kumar ${ }^{1}$, Amadou Hama ${ }^{2}$ and Anjan Biswas ${ }^{2,3, *}$ \\ ${ }^{1}$ Department of Applied Sciences, Bahra Group of Institutes Patiala-147001, Punjab, India \\ 2 Department of Mathematical Sciences, Delaware State University, Dover, DE 19901-2277, USA \\ ${ }^{3}$ Department of Mathematics, Faculty of Sciences, King Abdulaziz University, Jeddah-21589, Kingdom of Saudi Arabia
}

Received: 7 Jul. 2013, Revised: 9 Oct. 2013, Accepted: 10 Oct. 2013

Published online: 1 Jul. 2014

\begin{abstract}
In this paper, the Konopelchenko-Dubrovsky equation will be studied by the aid of traveling wave hypothesis and Lie symmetry analysis. The traveling wave hypothesis will extract the 1-soliton solution while the Lie symmetry approach will retrieve other solutions to this equation.
\end{abstract}

Keywords: Konopelchenko-Dubrovsky equation, traveling wave hypothesis, Lie symmetry analysis.

\section{Introduction}

In the past few decades a lot of study has been conducted in the area of nonlinear evolution equations (NLEEs) that arise in various areas of Applied Mathematics and Mathematical Physics. Finding the exact analytical solution has been an ongoing challenge for these NLEEs. There has been a lot of success in this direction. In fact, in the past decade, there has been a lot of analytical tools developed that integrated several of these NLEEs. Some of them are variational iteration method, homotopy analysis method, Riccati's equation method, tanh-coth method, sine-cosine method, exp-function method, $G^{\prime} / G$ method and several others. In this paper, the traveling wave hypothesis as well as the Lie symmetry analysis will be used to extract soliton and other solutions to the Konopelchenko-Dubrovsky (KD) equation.

\section{Mathematical analysis}

The dimensionless form of the KD equation is given by

$q_{t}+a q q_{x}+b q^{2} q_{x}+c q_{x x x}+\alpha r_{y}+\beta q_{x} r=0$

$q_{y}=r_{x}$

This is a coupled system of equation where the dependent variables are $q(x, y, t)$ and $r(x, y, t)$ which are real valued functions, while the independent variables are $x, y$ and $t$ which represent the spatial and temporal variables. The spatial variables are in $x$ and $y$ directions. The coefficients $a, b, c, \alpha$ and $\beta$ are real valued constant coefficients. KD equation arise in Mathematical Physics in the context of nonlinear waves with weak dispersion. This coupled system cover the Gardner, KP, modified KP (mKP) and $\mathrm{KD}$ equations, for different values of coefficients $a, b, c$, $\alpha$ and $\beta$. For $r=0$, system reduces to the Gardner's equation $[16,17]$. For $b=\beta=0$, system of equations (1) and (2) become the KP equations [18,19] which is used to model the shallow water waves with weakly nonlinear restoring forces and nonlinear waves in ferromagnetic media, plasmas and super-fluids. For $a=0$, system reduces to $\mathrm{mKP}$ equations [20] which can describe the propagation of ion-acoustic waves in a plasma with the non-isothermal electrons. For $a=-6 \gamma, c=-1$, $b=1.5 \delta^{2}, \alpha=-3$ and $\beta=3 \delta$, equations reduces to $\mathrm{KD}$ equations $[21,22]$ which have proposed by the inverse scattering transformation method. So investigation of coupled system (1) and (2) will be of certain interest. In this paper, this coupled system of equation will be studied by the traveling wave hypothesis and Lie symmetry approach in the following two subsections.

\footnotetext{
*Corresponding author e-mail: biswas.anjan@gmail.com
} 


\subsection{Traveling wave}

In order to solve equations (1) and (2) by traveling wave, the starting hypothesis is

$q(x, y, t)=g\left(B_{1} x+B_{2} y-v t\right)$,

$r(x, y, t)=h\left(B_{1} x+B_{2} y-v t\right)$

where $g$ and $h$ are the wave profiles and $v$ is the velocity of the wave. The parameters $B_{1}$ and $B_{2}$ represent the inverse width of the wave in the $x$ - and $y$-directions. Substituting these into (1) and (2) gives the following coupled system of ordinary differential equations (ODEs).

$$
\begin{aligned}
-v g^{\prime}+a B_{1} g g^{\prime}+b B_{1} g^{2} g^{\prime}+c B_{1}^{3} g^{\prime \prime \prime} & +\alpha B_{2} h^{\prime} \\
& +\beta B_{1} g^{\prime} h=0
\end{aligned}
$$

and

$B_{2} g^{\prime}=B_{1} h^{\prime}$

where $^{\prime}$ denotes differentiation with respect to $s$ and

$s=B_{1} x+B_{2} y-v t$

Integrating (5) and (6) once and eliminating $h$ between them while choosing the integration constant to be zero, since the search is for a soliton solution, gives the ODE in $g$ as

$g^{\prime \prime}=a_{1} g^{3}-a_{2} g^{2}+a_{3} g$

where

$a_{1}=-\frac{b}{3 c B_{1}}$

$a_{2}=\frac{a B_{1}+\beta B_{2}}{2 c B_{1}^{3}}$

and

$a_{3}=\frac{v B_{1}-\alpha B_{2}^{2}}{c B_{1}^{3}}$

Now, multiplying both sides of (9) by $g^{\prime}$ and integrating and once again choosing the integration constant to be zero yields

$\left(g^{\prime}\right)^{2}=\frac{g^{2}}{6}\left(3 a_{1} g^{2}-4 a_{2} g+6 a_{3}\right)$

Separating variables and integrating gives

$\frac{B_{1} x+B_{2} y-v t}{\sqrt{6}}=\frac{d s}{g \sqrt{3 a_{1} g^{2}-4 a_{2} g+6 a_{3}}}$

which, after simplification, leads to the soliton solution given by

$$
\begin{aligned}
q(x, y, t) & =g\left(B_{1} x+B_{2} y-v t\right) \\
& =\frac{A}{D+\cosh \left(B_{1} x+B_{2} y-v t\right)}
\end{aligned}
$$

where the amplitude $A$ of the solitary wave is given by given by

$A=\frac{6 a_{3}}{\sqrt{4 a_{2}^{2}-18 a_{1} a_{3}}}$

while the constant $D$ is

$D=\frac{18 a_{1} a_{2} a_{3}}{\left(2 a_{2}^{2}+9 a_{1} a_{3}\right) \sqrt{4 a_{2}^{2}-18 a_{1} a_{3}}}$.

These poses the constraint

$9 a_{1} a_{3}<2 a_{2}^{2}$

Finally, the wave profile for $r(x, y, t)$ is given by

$r(x, y, t)=h\left(B_{1} x+B_{2} y-v t\right)=\frac{B_{2}}{B_{1}} q(x, y, t)$

which is obtained from (6).

\subsection{Symmetry analysis}

In this subsection, we apply Lie symmetry approach [11, 12] to find symmetries and we obtain some exact solutions of equations (1) and (2).

\subsubsection{Lie symmetry approach}

In order to find the symmetry group of equations (1) and (2), we apply the algorithms described in text books such as [13-15]. Setting the one-parameter Lie group of infinitesimal transformations

$$
\begin{aligned}
& x \rightarrow \xi=x+\varepsilon \xi+O\left(\varepsilon^{2}\right) \\
& y \rightarrow \zeta=y+\varepsilon \zeta+O\left(\varepsilon^{2}\right) \\
& t \rightarrow \tau=t+\varepsilon \tau+O\left(\varepsilon^{2}\right) \\
& r \rightarrow \phi=r+\varepsilon \phi+O\left(\varepsilon^{2}\right) \\
& q \rightarrow \eta=q+\varepsilon \eta+O\left(\varepsilon^{2}\right),
\end{aligned}
$$

where the infinitesimals $\xi, \zeta, \tau, \phi, \eta$ depend on $x, y, t, r$, and $q$ in general, with small parameter $\varepsilon \ll 1$. We look for an algebra of vector fields of the form

$$
V=\xi \partial_{x}+\zeta \partial_{y}+\tau \partial_{t}+\phi \partial_{r}+\eta \partial_{q}
$$

The coefficients $\xi, \zeta, \tau, \phi, \eta$ are determined from the requirement that the third prolongation of $V$ should annihilate the equations on the solution set of the equations.

The invariance of equations (1) and (2) under the infinitesimal point transformations (19) leads to following expressions for infinitesimals

$$
\begin{aligned}
& \eta=-\frac{(a+2 q b)}{6 b} C_{1} \\
& \phi=\frac{f^{\prime}(t)}{\beta}+\frac{a^{2}-4 b \beta r}{6 b \beta} C_{1} \\
& \tau=C_{1} t+C_{2} \\
& \xi=\frac{x}{3} C_{1}+f(t) \\
& \zeta=\frac{2 y}{3} C_{1}+C_{3},
\end{aligned}
$$


where $f(t)$ is arbitrary function of $t$ and $C_{1}, C_{2}, C_{3}, C_{4}$ are arbitrary constants and prime $\left({ }^{\prime}\right)$ denotes derivative with respect to $t$. Associated with this Lie group, we have an infinite dimensional Lie algebra that can be represented by the generators

$$
\begin{aligned}
& V_{1}=-\frac{(a+2 q b)}{6 b} \frac{\partial}{\partial q}+\frac{a^{2}-4 b \beta r}{6 b \beta} \frac{\partial}{\partial r}+t \frac{\partial}{\partial t}+\frac{x}{3} \frac{\partial}{\partial x}+\frac{2 y}{3} \frac{\partial}{\partial y} \\
& V_{2}=\frac{\partial}{\partial t} \\
& V_{3}=\frac{\partial}{\partial y} \\
& V_{4}=\frac{f^{\prime}(t)}{\beta} \frac{\partial}{\partial r}+f(t) \frac{\partial}{\partial x} .
\end{aligned}
$$

\subsubsection{Similarity reductions and invariant solutions}

To obtain the symmetry reductions of equations (1) and (2), we have to solve the characteristic equation

$$
\frac{d x}{\xi}=\frac{d y}{\zeta}=\frac{d t}{\tau}=\frac{d r}{\phi}=\frac{d q}{\eta},
$$

where $\xi, \zeta, \tau, \phi$ and $\eta$ are given by (21). To solve (23), we consider three cases:

(i) $V_{2}+\mu V_{4},(i i) V_{3}+V_{4}$ and (iii) $V_{4}$

\section{Case (i) $V_{2}+\mu V_{4}$}

Solving characteristic equation (23), we have following similarity variables

$$
\begin{gathered}
\theta=y \\
\rho=x-\mu \int f(t) d t \\
q=F(\theta, \rho) \\
r=\frac{\mu}{\beta} f(t)+G(\theta, \rho),
\end{gathered}
$$

where $\theta, \rho$ are new independent variables and $F, G$ are new dependent variables. Substituting equation (25) along with equations (24) into equations (1) and (2), we immediately obtain the reduced equations, which read

$$
\begin{aligned}
& F_{\theta}=G_{\rho} \\
& c F_{\rho \rho \rho}+b F^{2} F_{\rho}+a F F_{\rho}+\beta G F_{\rho}+\alpha G_{\theta}=0 .
\end{aligned}
$$

Again apply Lie classical method on system (26), we obtain symmetries as follows:

$$
\begin{aligned}
& \tau_{1}=C_{1}+2 \theta C_{3} \\
& \tau_{2}=C_{2}+\rho C_{3} \\
& \phi^{1}=\frac{-2 b F-a}{2 b} C_{3} \\
& \eta^{1}=\frac{a^{2}-4 b \beta G}{2 \beta b} C_{3}
\end{aligned}
$$

where $\tau_{1}, \tau_{2}, \phi^{1}, \eta^{1}$ are infinitesimals corresponding to $\theta, \rho, F, G$, respectively. $C_{1}, C_{2}$ and $C_{3}$ are arbitrary constants. Three dimensional Lie algebra admitted by (26) is

$$
\begin{aligned}
L_{3}=\left[V_{1}=\frac{\partial}{\partial \theta}, V_{2}=\frac{\partial}{\partial \rho}\right. \\
\left.V_{3}=2 \theta \frac{\partial}{\partial \theta}+\rho \frac{\partial}{\partial \rho}-\frac{2 b F+a}{2 b} \frac{\partial}{\partial F}+\frac{a^{2}-4 b \beta G}{2 \beta b} \frac{\partial}{\partial G}\right]
\end{aligned}
$$

The problem of deriving an optimal system of group invariant solutions is equivalent to find an optimal system of Lie symmetries (or subalgebras spanned by these operators). The method used here is given by Olver in [14]. For brevity we omit the details, and just state the result that an optimal system of generators is

$$
\begin{aligned}
& \text { (i) } V_{3} \\
& \text { (ii) } V_{2}+l V_{1} \\
& \text { (iii) } V_{1},
\end{aligned}
$$

where $l$ is arbitrary constant.

We use the method of characteristics to determine the invariants and reduced ODEs corresponding to each subalgebra given in (29). Symmetry variables and the invariants of the subalgebras of the Lie algebra $L_{3}$ are given in table 1 .

Table 1: Similarity variables of equation (26)

\begin{tabular}{|c|c|c|}
\hline Subalgebra & Symmetry variables & Functions $F(\theta, \rho), G(\theta, \rho)$ \\
\hline$V_{3}$ & $\sigma=\frac{\rho^{2}}{\theta}$ & $F=\frac{H(\sigma)}{\rho}-\frac{a}{2 b}, G=\frac{J(\sigma)}{\rho^{2}}+\frac{a^{2}}{4 b \beta}$ \\
$V_{2}+l V_{1}$ & $\sigma=\theta-l \rho$ & $F=H(\sigma), G=J(\sigma)$ \\
$V_{1}$ & $\sigma=\rho$ & $F=H(\sigma), G=J(\sigma)$ \\
\hline
\end{tabular}

Now reduction of variable is performed to obtain ODE. Exact solutions of ODE are discussed in each case.

\section{Vector field $V_{3}$}

The reduced ODE system is

$$
\begin{array}{r}
-\sigma^{2} H^{\prime}-2 \sigma J^{\prime}+2 J=0 \\
-8 c \sigma^{3}-2 b \sigma H^{2} H^{\prime}+b H^{3}-6 c \sigma H^{\prime}+6 c H \\
+\alpha \sigma^{2} J^{\prime}-2 \beta \sigma H^{\prime} J+\beta H J=0,
\end{array}
$$

where $\left({ }^{\prime}\right)$ denotes $\sigma$-derivative.

We obtain following solution of ODE system (30)

$$
H=k_{1} \sigma, J=-\frac{k_{1}}{2} \sigma^{2}
$$

where

$$
k_{1}=\frac{\beta \pm \sqrt{\beta^{2}-16 b \alpha}}{4 b} .
$$

Corresponding solution of main equations (1) and (2) is given as

$$
\begin{aligned}
& q(x, y, t)=k_{1} \frac{x-\mu \int f(t) d t}{y}-\frac{a}{b} \\
& r(x, y, t)=\frac{\mu}{\beta} f(t)-k_{1} \frac{\left(x-\mu \int f(t) d t\right)^{2}}{2 y^{2}}+\frac{a^{2}}{4 b \beta}
\end{aligned}
$$


where $k_{1}$ is given by (32).

Vector field $V_{2}+l V_{1}$

Corresponding reduced ODE system is

$$
\begin{aligned}
l^{3} c H^{\prime \prime \prime}+l b H^{2} H^{\prime}+l a H H^{\prime}+l \beta H^{\prime} J-\alpha J^{\prime} & =0 \\
H^{\prime}+l J^{\prime} & =0,
\end{aligned}
$$

where prime denotes derivative with respect to $\sigma$. Integrating (35) with respect to $\sigma$, we have

$$
J=-\frac{H}{l}+C_{1}
$$

where $C_{1}$ is arbitrary constant.

Using (35)-(36) in (34) and integrating twice with respect to $\sigma$, we have

$$
\begin{aligned}
& 6 l^{4} c H^{\prime 2}+l^{2} b H^{4}+2 l(a l-\beta) H^{3}+6\left(\alpha+C_{1} l^{2} \beta\right) H^{2} \\
& +C_{2} H+C_{3}=0
\end{aligned}
$$

where $C_{2}$ and $C_{3}$ are arbitrary constants. Taking

$$
l=\frac{\beta}{a}, C_{2}=C_{3}=0
$$

and substituting

$$
H(\sigma)^{2}=X(\sigma)
$$

in (37), we have

$$
3 c l^{4} X^{\prime 2}+2 b l^{2} X^{3}+12\left(\alpha+C_{1} \beta l^{2}\right) X^{2}=0 .
$$

Solutions of equation (39) are as follows:

$$
\begin{aligned}
\text { (i) } X(\sigma)= & -6 \frac{\left(\alpha+C_{1} \beta l^{2}\right)}{l^{2} b} \operatorname{sech}^{2}\left(C_{4}+\frac{\sqrt{-c\left(\alpha+C_{1} \beta l^{2}\right)} \sigma}{c l^{2}}\right) \\
\text { (ii) } X(\sigma)= & -6 \frac{\left(\alpha+C_{1} \beta l^{2}\right)}{l^{2} b} \csc ^{2}\left(C_{4}-\frac{\sqrt{c\left(\alpha+C_{1} \beta l^{2}\right)} \sigma}{c l^{2}}\right) \\
(\text { iii } X(\sigma)= & -2 \frac{\alpha+C_{1} \beta l^{2}}{l^{2} b} \\
& -6 C_{4}{ }^{2} \frac{c l^{2}}{b} \wp\left(C_{5}+C_{4} \sigma, m_{1}, m_{2}\right),
\end{aligned}
$$

where

$$
\begin{aligned}
l & =\frac{\beta}{a}, m_{1}=\frac{4}{3} \frac{\left(\alpha^{2}+2 \alpha C_{1} \beta l^{2}+C_{1}^{2} \beta^{2} l^{4}\right)}{C_{4}{ }^{4} c^{2} l^{8}}, \\
m_{2} & =\frac{8}{27} \frac{\left(\alpha+C_{1} \beta l^{2}\right)\left(\alpha^{2}+2 \alpha C_{1} \beta l^{2}+C_{1}{ }^{2} \beta^{2} l^{4}\right)}{l^{12} c^{3} C_{4}{ }^{6}}
\end{aligned}
$$

and $C_{4}, C_{5}$ are arbitrary constants. $\wp$ is Weierstrass $\mathrm{P}$ function.
Corresponding solutions of main system of equations (1) and (2) are

$$
\begin{aligned}
\text { (i) } q(x, y, t)= & \frac{a}{\beta} \sqrt{-\frac{6}{b} k_{1} \operatorname{sech}\left(C_{4}+\frac{a^{2}}{c \beta^{2}} \sqrt{-c k_{1}}\right.} \\
& \left.\left(y-\frac{\beta\left(x-\mu \int f(t) d t\right)}{a}\right)\right) \\
r(x, y, t)= & -\frac{a^{2}}{\beta^{2}} \sqrt{-\frac{6}{b} k_{1} \operatorname{sech}\left(C_{4}+\frac{a^{2}}{c \beta^{2}} \sqrt{-c k_{1}}\right.} \\
& \left.\left(y-\frac{\beta\left(x-\mu \int f(t) d t\right)}{a}\right)\right) \\
& +\frac{f(t) \mu}{\beta}+C_{1}
\end{aligned}
$$

(ii) $q(x, y, t)=\frac{a}{\beta} \sqrt{-\frac{6}{b} k_{1}} \csc \left(C_{4}-\frac{a^{2}}{c \beta^{2}} \sqrt{c k_{1}}\right.$

$$
\left.\left(y-\frac{\beta\left(x-\mu \int f(t) d t\right)}{a}\right)\right)
$$

$$
\begin{aligned}
r(x, y, t)= & -\frac{a^{2}}{\beta^{2}} \sqrt{-\frac{6}{b} k_{1}} \csc \left(C_{4}-\frac{a^{2}}{c \beta^{2}} \sqrt{c k_{1}}\right. \\
& \left.\left(y-\frac{\beta\left(x-\mu \int f(t) d t\right)}{a}\right)\right) \\
& +\frac{f(t) \mu}{\beta}+C_{1}
\end{aligned}
$$

(iii) $q(x, y, t)=\sqrt{-\frac{2 k_{1} a^{2}}{\beta^{2} b}} \cdots$

$$
\begin{aligned}
& \cdots-6 \frac{C_{4}^{2} c \beta^{2}}{a^{2} b} \wp\left(C_{5}+C_{4} \sigma, m_{1}, m_{2}\right) \\
r(x, y, t)= & -\frac{a}{\beta} \sqrt{-\frac{2 k_{1} a^{2}}{\beta^{2} b} \cdots} \\
& \cdots-6 \frac{C_{4}^{2} c \beta^{2}}{a^{2} b} \wp\left(C_{5}+C_{4} \sigma, m_{1}, m_{2}\right) \\
& +\frac{f(t) \mu}{\beta}+C_{1},
\end{aligned}
$$

where $\sigma=\left(y-\frac{\beta\left(x-\mu \int f(t) d t\right)}{a}\right), \quad k_{1}=\alpha+\frac{C_{1} \beta^{3}}{a^{2}}$ and $m_{1}, m_{2}$ is given by (41).

Vector field $V_{1}$

Corresponding reduced ODE system is

$$
\begin{aligned}
J^{\prime} & =0 \\
c H^{\prime \prime \prime}+b H^{2} H^{\prime}+a H H^{\prime}+\beta J H^{\prime} & =0,
\end{aligned}
$$

where prime denotes derivative with respect to $\sigma$. 
Consequently, solution of system of equations (1) and (2) is given as

$$
\begin{aligned}
q= & -\frac{1}{2} \frac{a}{b}-\frac{1}{2 b} \sqrt{-12 b \beta k+3 a^{2}} \\
& \tanh \left(\frac{\sqrt{-2 b c\left(-4 b \beta k+a^{2}\right)}\left(x-\mu \int f(t) d t\right)}{4 b c}\right) \\
r= & \frac{\mu f(t)}{\beta}+k,
\end{aligned}
$$

where $k$ is arbitrary constant.

$$
\text { Case (ii) } V_{3}+V_{4}
$$

Corresponding similarity variables are

$$
\begin{aligned}
& \rho=x-f(t) y \\
& \theta=t \\
& q=F(\theta, \rho) \\
& r=\frac{\dot{f}}{\beta} y+G(\theta, \rho),
\end{aligned}
$$

where $\rho, \theta$ are new independent variables and $F, G$ are new dependent variables. Dot denotes t-derivative. Using (47) in system of equations (1) and (2), we have

$$
\begin{array}{r}
f(\theta) F_{\rho}+G_{\rho}=0 \\
-c \beta F_{\rho \rho \rho}-b \beta F^{2} F_{\rho}-a \beta F F_{\rho}-\beta F_{\theta} \\
+\alpha \beta f(\theta) G_{\rho}-\beta^{2} F_{\rho} G-\alpha \frac{d f}{d \theta}=0
\end{array}
$$

Integrating (48) with respect to $\rho$ and taking function of integration equal to zero, we have

$G=-f(\theta) F$

Using (48) and (50) in (49), we have

$$
\begin{aligned}
& -c \beta F_{\rho \rho \rho}-b \beta F^{2} F_{\rho}-a \beta F F_{\rho}-\beta F_{\theta}-\alpha \beta f^{2}(\theta) F_{\tau} \\
& +\beta^{2} f(\theta) F F_{\rho}-\alpha \frac{d f}{d \theta}=0,
\end{aligned}
$$

which is difficult to solve for general $f(\theta)$. So to simplify equation (51) we take $f(\theta)=\frac{a}{\beta}$, so that, equation (51) reduces to

$c \beta F_{\rho \rho \rho}+b \beta F^{2} F_{\rho}+\beta F_{\theta}+\frac{a^{2} \alpha}{\beta} F_{\tau}=0$,

Again applying Lie classical method on (52), we obtain following infinitesimals

$$
\begin{aligned}
\tau_{1} & =\theta C_{1}+C_{2} \\
\tau_{2} & =\left(\frac{\rho}{3}+\frac{2 a^{2} \alpha \theta}{3 \beta^{2}}\right) C_{1}+C_{3} \\
\eta^{1} & =-\frac{F}{3} C_{1},
\end{aligned}
$$

where $\tau_{1}, \tau_{2}$ and $\eta^{1}$ are infinitesimals corresponding to $\theta, \rho$ and $F$, respectively. Corresponding vector fields are given as

$$
\begin{aligned}
& V_{1}=\theta \frac{\partial}{\partial \theta}+\left(\frac{\rho}{3}+\frac{2 a^{2} \alpha \theta}{3 \beta^{2}}\right) \frac{\partial}{\partial \rho}-\frac{F}{3} \frac{\partial}{\partial F} \\
& V_{2}=\frac{\partial}{\partial \theta} \\
& V_{3}=\frac{\partial}{\partial \rho} .
\end{aligned}
$$

Following the same way as in Case (i), similarity variables and invariant functions of (54) are given in Table 2.

Table 2: Similarity variables of equation (52)

\begin{tabular}{|c|c|c|}
\hline Subalgebra & Symmetry variables & Function $F(\theta, \rho)$ \\
\hline$V_{1}$ & $\sigma=\rho \theta^{-\frac{1}{3}}-\frac{a^{2} \alpha}{\beta^{2}} \theta^{\frac{2}{3}}$ & $F=\theta^{-\frac{1}{3}} H(\sigma)$ \\
$V_{2}+\lambda V_{3}$ & $\sigma=\rho-\lambda \theta$ & $F=H(\sigma)$ \\
$V_{3}$ & $\sigma=\theta$ & $F=H(\sigma)$ \\
\hline
\end{tabular}

Vector field $V_{1}$

The reduced ODE is

$$
3 c H^{\prime \prime \prime}+3 b H^{2} H^{\prime}-(H \sigma)^{\prime}=0,
$$

where prime denotes derivative with respect to $\sigma$. Solution of ODE (55) is given as

$$
H(\sigma)= \pm \frac{\sqrt{-\frac{6 c}{b}}}{\sigma}
$$

Consequently, solution of main system of equations (1) and (2) is given as

$$
\begin{array}{r}
q= \pm \frac{\sqrt{-\frac{6 c}{b}}}{x+\frac{a}{\beta} y-\frac{a^{2} \alpha}{\beta} t} \\
r=\mp \frac{a}{\beta} \frac{\sqrt{-\frac{6 c}{b}}}{x+\frac{a}{\beta} y-\frac{a^{2} \alpha}{\beta} t} .
\end{array}
$$

Vector field $V_{2}+\lambda V_{3}$ The reduced ODE is

$$
c \beta H^{\prime \prime \prime}+b \beta H^{2} H^{\prime}+\left(\frac{\alpha a^{2}}{\beta}-\lambda \beta\right) H^{\prime}=0 .
$$


Solutions of (59) are given as

$$
\begin{aligned}
\text { (i) } H(\sigma)= & \pm \frac{\sqrt{-3 b\left(-c \beta^{2} C_{3}^{2}+\alpha a^{2}-\lambda \beta^{2}\right)}}{b \beta} \\
& \operatorname{cn}\left(C_{2}+C_{3} \sigma, n_{1}\right) \\
(\text { ii }) H(\sigma)= & \pm \frac{\sqrt{6 b c} C_{3}}{b} \operatorname{dn}\left(C_{2}+C_{3} \sigma, n_{2}\right) \\
\text { (iii) } H(\sigma)= & \frac{\sqrt{3 b\left(\alpha a^{2}-\lambda \beta^{2}\right)}}{b \beta} \\
& \tan \left(C_{1}+\frac{\sqrt{-\left(2 \alpha a^{2}-2 \lambda \beta^{2}\right) c} \sigma}{2 c \beta}\right)
\end{aligned}
$$

where

$$
\begin{aligned}
& n_{1}=\frac{\sqrt{-2 c\left(-c \beta^{2} C_{3}^{2}+\alpha a^{2}-\lambda \beta^{2}\right)}}{2 c \beta C_{3}}, \\
& n_{2}=\frac{\sqrt{c\left(2 c \beta^{2} C_{3}^{2}+\alpha a^{2}-\lambda \beta^{2}\right)}}{c \beta C_{3}}
\end{aligned}
$$

and $C_{1}, C_{2}, C_{3}$ are arbitrary constants.

Consequently, solutions of $\mathrm{KD}$ equation is given as

$$
\begin{aligned}
& \text { (i) } q(x, y, t)= \pm \frac{\sqrt{-3 b\left(-c \beta^{2} C_{3}^{2}+\alpha a^{2}-\lambda \beta^{2}\right)}}{b \beta} \\
& \text { cn }\left(C_{2}+C_{3}\left(x+\frac{a}{\beta} y-\lambda t\right), n_{1}\right) \\
& r(x, y, t)= \pm \frac{a}{\beta} \frac{\sqrt{-3 b\left(-c \beta^{2} C_{3}^{2}+\alpha a^{2}-\lambda \beta^{2}\right)}}{b \beta} \\
& \text { cn }\left(C_{2}+C_{3}\left(x+\frac{a}{\beta} y-\lambda t\right), n_{1}\right) \\
& \text { (ii) } q(x, y, t)= \pm \frac{\sqrt{6 b c} C_{3}}{b} \\
& \operatorname{dn}\left(C_{2}+C_{3}\left(x+\frac{a}{\beta} y-\lambda t\right), n_{2}\right) \\
& r(x, y, t)= \pm \frac{a}{\beta} \frac{\sqrt{6 b c} C_{3}}{b} \\
& \operatorname{dn}\left(C_{2}+C_{3}\left(x+\frac{a}{\beta} y-\lambda t\right), n_{2}\right) \\
& \text { (iii) } q(x, y, t)=\frac{\sqrt{3 b\left(\alpha a^{2}-\lambda \beta^{2}\right)}}{b \beta} \\
& \tan \left(C_{1}+\frac{\sqrt{-\left(2 \alpha a^{2}-2 \lambda \beta^{2}\right) c}\left(x+\frac{a}{\beta} y-\lambda t\right)}{2 c \beta}\right) \\
& r(x, y, t)=\frac{a}{\beta} \frac{\sqrt{3 b\left(\alpha a^{2}-\lambda \beta^{2}\right)}}{b \beta} \\
& \tan \left(C_{1}+\frac{\sqrt{-\left(2 \alpha a^{2}-2 \lambda \beta^{2}\right) c}\left(x+\frac{a}{\beta} y-\lambda t\right)}{2 c \beta}\right)
\end{aligned}
$$

Vector field $V_{3}$

Corresponding to this vector field we get constant solution of KD equations (1) and (2).

\section{Case (iii) $V_{4}$}

Corresponding similarity variables are

$\theta=y$

$\rho=t$

$u=F(\theta, \rho)$

$v=\frac{\dot{f}}{\beta f} x+G(\theta, \rho)$,

where dot denotes derivative with respect to $t$. $\theta, \rho$ are new independent variables and $F, G$ are new dependent variables. Using (63) in $\mathrm{KD}$ equations (1) and (2), we have following two dimensional PDE

$$
\begin{gathered}
F_{\theta}-\frac{\frac{d f(\rho)}{d \rho}}{\beta f(\rho)}=0 \\
F_{\rho}+\alpha G_{\theta}=0 .
\end{gathered}
$$

General solution of equations (64) and (65) is

$$
\begin{aligned}
& F(\theta, \rho)=\frac{\frac{d f(\rho)}{d \rho}}{\beta f(\rho)} \theta+g(\rho) \\
& G(\theta, \rho)=-\frac{f(\rho) \frac{d^{2} f(\rho)}{d \rho^{2}}}{\alpha \beta f^{2}(\rho)} \frac{\theta^{2}}{2}-\frac{d g(\rho)}{d \rho} \frac{\theta}{\alpha}-h(\rho),
\end{aligned}
$$

where $f, g, h$ are arbitrary functions of $\rho$. Consequently, solution of KD equation is

$q=\frac{\dot{f}}{\beta f} y+g(t)$

$r=\frac{\dot{f}}{\beta f} x-\frac{f \ddot{f}-\dot{f}^{2}}{\alpha \beta f^{2}} \frac{y^{2}}{2}-\frac{y}{\alpha} \dot{g}-h(t)$,

where dot denotes derivative with respect to $t$ and $f, g, h$ are arbitrary functions of $t$.

\section{Conclusions}

This paper shined some light on the KD equation from the perspective of its integrability. The traveling wave hypothesis was applied to retrieve a solitary wave solution to the equation. Subsequently, a much more powerful integration tool was implemented to extract more solutions to the equation. This is the Lie symmetry analysis. This second tool retrieved solitons, periodic singular waves as well as cnoidal and snoidal waves. These solutions are going to be immensely helpful in further future analysis to this equation.

The future research for this equation holds on a strong footing. The perturbation terms will be added and the 
quasi stationary soliton solutions will be obtained. Additionally, the stochastic perturbation term will be added and the corresponding mean free velocity of the solitons will be retrieved after formulating the corresponding Langevin equation. The equation will be further analyzed with time dependent coefficients. These form just the tip of the iceberg.

Remarks 3.1 We feel worth mentioning that the solutions (33), (42), (43), (44), (46) and (68) obtained are such that one can choose the arbitrary function $f(t)$ in a suitable manner to obtain variety of solutions of KD equation.

\section{References}

[1] B. Cao, Acta Appl. Math. 112, 181 (2010).

[2] L Ji, L Sen-Yue and W Ke-Lin, Chinese Phys. Lett. 18, 1173 (2001).

[3] B. Li and Y. Zhang, Chaos Solitons Fractals 38, 1202 (2008).

[4] S. Li-Na and Z. Hong-Qing, Comm. Theoret. Phys. 45, 769 (2006).

[5] N. Taghizadeh and M. Mirzazadeh, Appl. Appl. Math. 6, 1893 (2011).

[6] Y. Wang and L. Wei, Chaos Solitons Fractals 15216 (2010).

[7] A.M. Wazwaz, J. Nat. Sci. Math. 1, 1 (2007).

[8] Y. Zhang and S. Lai, Dynam. Contin. Discrete Impuls. Systems 16, 559 (2009).

[9] H. Zhao, J.G. Han, W.T. Wang and H.Y. An, Czechoslovak J. Phys. 56, 1381 (2006).

[10] L. Zhi-Fang and R. Hang-Yu, Comm. Theoret. Phys. 44, 385 (2005).

[11] K. Singh, R.K. Gupta and S. Kumar, Appl. Math. Comput. 17, 7021 (2011).

[12] S. Kumar, K. Singh and R.K. Gupta, Commun. Nonlinear Sci. Numer. Simul. 17, 1529 (2012).

[13] L.V. Ovsiannikov, Group Properties of Differential Equations (Novosibirsk, Moscow, 1962).

[14] P.J. Olver, Applications of Lie Groups to Differential Equations (Springer-Verlag, New York, Heidelberg, Berlin, Tokyo, 1993).

[15] G.W. Bluman and J.D. Cole, Similarity Methods for Differential Equations (Springer-Verlag, New York, Heidelberg, Berlin, 1974).

[16] E.V. Krishnan, H. Triki, M. Labidi and A. Biswas, Nonlinear Dyn. 66, 497 (2011).

[17] X.G. Xu, X.H. Meng, Y.T. Gao and X.Y. Wen, Appl. Math. Comput. 210, 313 (2009).

[18] A.M.Wazwaz, Appl.Math. Comput. 190, 633 (2007).

[19] C. M. Khalique \& A. R. Adem. Appl. Math. Comput. Volume 216, 2849. (2010).

[20] Z.Y. Sun, Y.T. Gao, X. Yu, X.H. Meng and Y. Liu, Wave Motion 46, 511 (2009).

[21] B.G. Konopelcheno and V.G. Dubrovsky, Phys. Lett. A 102, 15 (1984).

[22] H.Y. Zhi, Appl.Math. Comput. 210, 530 (2009).

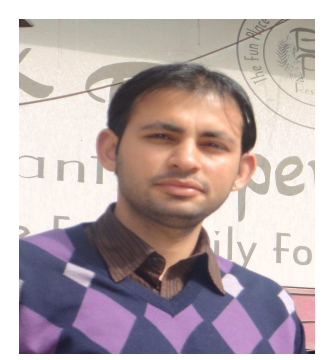

Sachin Kumar is
a postdoctoral fellow
in the Department of Mathematics at Panjab University, Chandigarh, India. He received his Ph.D from Thapar University, Patiala, India. His area of interest is symmetries and exact solutions of nonlinear partial differential equations. He has published a number of research papers in peer reviewed journals.

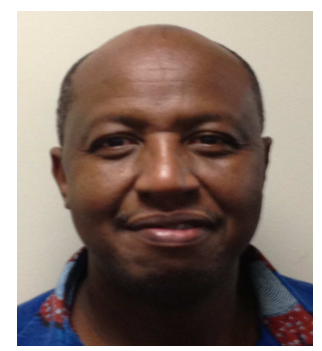

Amadou Hama Amadou Hama earned his B.S degree in Mathematics from University of Jos in Nigeria. Subsequently he obtained his M.Sc degree in Mathematics from University of Jos and Louisiana State University, USA. Currently, he is enrolled in $\mathrm{Ph} . \mathrm{D}$ program at Delaware State University, USA. His research area of interest is Theory of Solitons.

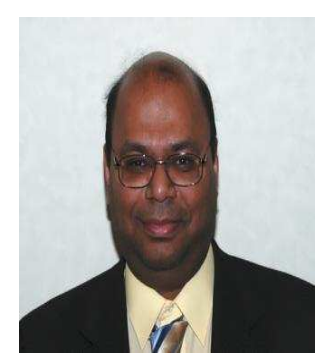

$\begin{gathered}\text { Anjan Biswas earned } \\ \text { his B.Sc (hons) degree } \\ \text { in Mathematics from }\end{gathered}$
St. Xavier's College in
Calcutta, India. Subsequently,
he earned his M.Sc and
M.Phil degrees from the
University of Calcutta. After
that he further earned his MA
and Ph.D. degrees from the University of New Mexico in Albuquerque, NM, USA. Currently, he is an Associate Professor in the Department of Mathematical Sciences at Delaware State University in Dover, DE. His research interest is on Theory of Solitons applicable to Physics, Engineering and Biosciences. 